\title{
Detection of fluoroquinolone residues in milk using Yersinia spp. strains: towards better sensitivity for flumequine determination
}

\author{
Pavlína Navrátilová, Jana Vyhnálková, Lenka Vorlová \\ Department of Milk Hygiene and Technology, Faculty of Veterinary Hygiene and Ecology, \\ University of Veterinary and Pharmaceutical Sciences Brno, 61242 Brno, Czech Republic \\ navratilovap@vfu.cz
}

Received: June 12, $2017 \quad$ Accepted: December 4, 2017

\begin{abstract}
Introduction: Plate diffusion methods play an important role in the monitoring system for antimicrobial agents in raw materials and foodstuffs of animal origin. The aim of this work was to select a Yersinia spp. strain for the plate diffusion method based on sensitivity to a fluoroquinolone, namely flumequine. Another aim was to determine the optimal conditions of the method with the selected strain of Yersinia ruckeri CCM 8467 and to determine the detection capability $(C C \beta)$ of this method for residues of selected fluoroquinolones in milk. Material and Methods: Optimum method conditions were set: cell concentration in the test agar at the level of $9.10^{5}-10^{6} \mathrm{CFU} / \mathrm{mL}$, discs with a diameter of $12.7 \mathrm{~mm}$, Antimicrobial Inhibitor Test Agar with a pH of 6.0, and incubation at $30^{\circ} \mathrm{C}$ for at least $18 \mathrm{~h}$ and up to $24 \mathrm{~h}$. Results: With respect to the maximum residue limit (MRL), the Y. ruckeri plate method demonstrated the lowest sensitivity to flumequine. The $C C \beta$ of the method for flumequine was in the concentration of $100 \mu \mathrm{g} / \mathrm{L}^{-1}$ (twice the MRL). The study also confirmed that the method exhibits very good sensitivity to the other tested fluoroquinolones, which were marbofloxacin (30 $\mu \mathrm{g} / \mathrm{L}, 0.4 \mathrm{MRL})$, ciprofloxacin $(10 \mu \mathrm{g} / \mathrm{L}, 0.1 \mathrm{MRL})$, and enrofloxacin $(20 \mu \mathrm{g} / \mathrm{L}, 0.2 \mathrm{MRL})$, but lower sensitivity to danofloxacin $(42 \mu \mathrm{g} / \mathrm{L}, 1.4 \mathrm{MRL})$. Conclusion: The method with the CCM 8467 strain of Y. ruckeri showed a higher sensitivity to flumequine than the method with the ATCC 11303 strain of E. coli.
\end{abstract}

Keywords: milk, quinolones, residues, plate diffusion method, Yersinia ruckeri.

\section{Introduction}

From the perspective of the quality and hygiene standard of milk, veterinary medicines are classified among foreign substances and contaminants. The presence of their residues in raw milk, especially if they are present in concentrations exceeding the permitted limits, is an issue of concern for public health. To protect the health of consumers while ensuring quality of raw materials for the production of dairy products, it is necessary to monitor the presence of veterinary drug residues in raw milk (6).

The monitoring system for veterinary medicine residues in animal products includes plate diffusion methods. They belong to the microbial inhibition methods and they are based on the reaction between a specific sensitive bacterial strain and an antimicrobial compound present in the sample examined (7). The advantages of these methods include the capability to simultaneously detect a broad spectrum of antimicrobials with different chemical structures. These techniques allow identification of groups of antimicrobials and their semi-quantitative determination, thus they are used as screening as well as post-screening methods $(6,15)$. Plate diffusion methods are being developed constantly. One of the main reasons for diversification is the everexpanding range of veterinary medicinal products approved for the treatment of animals intended for food production (10). The continuously adjusted requirements regarding analytical methods including those for antimicrobial detection in raw materials and foodstuffs of animal origin constitute another reason. Performance criteria and procedures for the validation of screening and confirmatory methods are established in the EU legislation (8).

Numerous studies on the development and validation of new plate diffusion methods published in the last twenty years aimed at increasing the sensitivity of the methods to selected analytes, due to the establishment of permissible antimicrobial limits (15, 
$18,21,22)$. Some of the studies are devoted to determining sensitivity of plate diffusion methods to a broad spectrum of antimicrobials. Other studies, however, focus on the development and validation of new methods aimed at detection of a specific group of antimicrobials. One of the antimicrobial groups which was the subject of interest of certain studies was quinolone chemotherapeutics.

Quinolone chemotherapeutics represent further progress in developing and expanding the spectrum of antimicrobials for effective treatment of infectious diseases in human medicine. Use of fluoroquinolones in veterinary medicine increased significantly at the end of the $20^{\text {th }}$ century globally, including in the Czech Republic. Their application varies depending on the animal species, indication, region, and permitted MRLs. As indicated by statistical data, the most commonly used agents in the treatment of livestock in Europe include enrofloxacin, difloxacin, flumequine, marbofloxacin, danofloxacin, and oxolinic acid (2).

To determine quinolone chemotherapeutic content, the plate diffusion method using an E. coli strain was added to the existing range of laboratory techniques, as recommended by Ellerbroek (11). The E. coli method is contained in a number of multiplate methods $(13,15,16$, 19). Some studies, however, confirmed that this method has a lower sensitivity to flumequine and oxolinic acid, and thus other bacteria sensitive to quinolones were tried. Alternatives to E. coli used in foreign studies to detect quinolone chemotherapeutics included Klebsiella pneumoniae ATTC 10031 and Yersinia ruckeri NCIMB 13282 (23). Nevertheless, most studies focused on increasing the sensitivity of plate methods to detect quinolone residues in muscles and organs, not in raw milk, although it is a strategic raw material for human nutrition, especially for children.

The aim of the study was to select a Yersinia spp. strain for the plate diffusion method based on sensitivity to flumequine, one of the fluoroquinolones. Another aim of the study was to set the optimum conditions of the plate method with the selected strain and to determine its sensitivity to selected fluoroquinolones. Fluoroquinolones used in veterinary medicine in cattle, i.e. danofloxacin, marbofloxacin, ciprofloxacin, enrofloxacin, and flumequine were selected for the study.

\section{Material and Methods}

Preparation of standard solutions. To prepare standard solutions of quinolone chemotherapeutics, the following analytical standards purchased from Sigma Aldrich (USA) were used: ciprofloxacin (17850, Fluka, Germany), enrofloxacin (17849, Fluka), flumequine (F 7016, Sigma Aldrich, USA), danofloxacin (3377, Riedel-deHaën, Germany), and marbofloxacin (34039, Riedel-deHaën). Standard solutions of fluoroquinolone chemotherapeutics with the active substance concentration of $c=1 \mathrm{mg} / \mathrm{mL}$ were prepared by initially dissolving a calculated weighed amount of the standard in $1 \mathrm{~mL}$ of
$0.1 \mathrm{~mol} / \mathrm{L} \mathrm{NaOH}$, and subsequently they were diluted to the desired volume with distilled water. Working solutions were prepared from standard solutions by further diluting with distilled water.

Tested strains. Strains of Yersinia spp. were obtained from the Czech Collection of Microorganisms (Czech Republic). The following strains of Yersinia spp. were used for the study: Y. aldovae CCM 3754 (4), Y. ruckeri CCM 4620 (12), Y. bercovieri CCM 4207 (24), Y. mollaretii CCM 4209 (24), Y. rohdei CCM 4075 (1), and Y. ruckeri (NCIMB 13 282) CCM 8467 (23).

Sensitivity testing with strains of Yersinia spp. and optimisation of conditions. Gelatin carriers of selected strains of Yersinia spp. were revived according to the manufacturer's recommendations (Czech Collection of Microorganisms). The 24-hour-old cultures in slant TSA (TSA, M593, HiMedia, India) were used to prepare test strain suspensions of various densities. Suspension density (CFU/mL) was determined by comparison with the McFarland standard $(0.5,1,2,3$, and 4.5$)$ and then checked by densitometer. The suspension was inoculated onto the test agar at $\mathrm{pH}$ 6.0 (Antimicrobial Inhibitor Test Agar with a $\mathrm{pH}$ of 6.0; M1631, HiMedia) and its $\mathrm{pH} 8.0$ counterpart (Antimicrobial Inhibitor Test Agar with a $\mathrm{pH}$ of 8.0; M1632 HiMedia) at $45-48^{\circ} \mathrm{C}$. Portions of the agar $(4 \mathrm{~mL})$ were transferred into prewarmed sterile glass Petri dishes of $90 \mathrm{~mm}$ diameter. Sensitivity of the methods to ciprofloxacin was tested using $10 \mu \mathrm{L}$ of working solution of ciprofloxacin $(c=0.3 \mu \mathrm{g} / \mathrm{mL})$ in a disc with a diameter of $6 \mathrm{~mm}$. Plates were incubated at the optimal strain growth temperature (according to the manufacturer's recommendations) for 18-24 h. Furthermore, the flumequine sensitivity of the plates with individual testing strains was tested with milk samples with known concentrations of flumequine (concentrations of $0.5,1,1.5,2,2.5$, and 3 MRL) by applying a $100 \mu \mathrm{L}$ sample to a $12.7 \mathrm{~mm}$ diameter disc.

Setting the optimum method conditions for the selected strain of $Y$. ruckeri CCM 8467. To adjust the method sensitivity, it was important to select the optimum agar $\mathrm{pH}$, the concentration of strain cells in agar (test strain suspension density), and incubation temperature $\left(30\right.$ or $\left.37^{\circ} \mathrm{C}\right)$. The method was tested with agar with $\mathrm{pH} 6.0$ and 8.0 and with various concentrations of strain cells $(\mathrm{CFU} / \mathrm{mL})$. Strain suspensions with various test strain concentrations were prepared using the McFarland scale $(0.5,1,2,3$, and 4.5). Setting the optimum method conditions was performed by testing milk samples with flumequine concentrations at levels of 0.5-3 times the MRL and by a procedure equivalent to sensitivity testing using ciprofloxacin solution.

Preparation of plate method with the test strain of $Y$. ruckeri CCM 8467. The strain of $Y$. ruckeri CCM 8467 supplied as a gelatin disc was revived in accordance with the producer's recommendations (Czech Collection of Microorganisms). The 24-hour-old culture in slant TSA (TSA, M593, HiMedia, India) was used to prepare the test strain suspension. Suspension concentration (amount of $\mathrm{CFU} / \mathrm{mL}$ ) was determined by 
comparison with the McFarland scale (no. 3). The suspension was inoculated in the test agar specified to pH 6.0 (Antimicrobial Inhibitor Test Agar, M1631, HiMedia) at $45-48^{\circ} \mathrm{C}$. Four millilitres of the agar was transferred into pre-warmed sterile glass Petri dishes of $90 \mathrm{~mm}$ diameter. Method sensitivity was tested using $10 \mu \mathrm{L}$ of ciprofloxacin working solution $(c=0.3 \mu \mathrm{g} / \mathrm{mL})$ in a 6-mm disc.

Preparation of fortified milk samples. Sensitivity of the plate diffusion method with Yersinia ruckeri CCM 8467 to selected fluoroquinolones was tested using samples of milk fortified with fluoroquinolones. Fortified milk samples were prepared by diluting a calculated amount of the working solution of chemotherapeutics in milk. Fortified milk samples with fluoroquinolone concentrations of $0.5,1,2,2.5$, and $3 \mathrm{MRL}$ were prepared from working solutions.

Determination of method sensitivity (detection capability). Sensitivity determination of the plate method with $Y$. ruckeri to the selected fluoroquinolones was performed using discs with a diameter of $12.7 \mathrm{~mm}$ (Blank Paper Discs, Albet LabScience, Spain) and absorbency of $100 \mu \mathrm{L}$ of milk sample. Each sample with the standard concentration of the antibiotic was tested 20 times. Plates were incubated at $30^{\circ} \mathrm{C}$ for at least $18 \mathrm{~h}$. After the incubation was complete, the size of the inhibition zone was evaluated. The zone size was measured using a dedicated scale (Antibiotic Zonescale, HiMedia). A regular inhibition zone around the disc of $\geq 2 \mathrm{~mm}$ was interpreted as a positive (suspect) test result.

\section{Results}

Testing sensitivity of collection strains to ciprofloxacin and flumequine. Selected collection strains of Yersinia spp., namely Y. aldovae CCM 3754 (4), Y. ruckeri CCM 4620 (12), Y. bercovieri CCM 4207 (24), Y. mollaretii CCM 4209 (24), Y. rohdei CCM 4075 (1), and Y. ruckeri CCM 8467 (23), were used as test strains in the plate diffusion method. Based on the strains' growth in agar media of different $\mathrm{pH}$ ( $\mathrm{pH} 6.0$ and 8.0 ), readability of the inhibition zones, and size of the inhibition zone around the disc with positive control, the optimal concentration of cells in the agar medium was set at about $9.10^{5}-10^{6} \mathrm{CFU} / \mathrm{mL}$ and the agar $\mathrm{pH}$ level of 6.0 was chosen. The plates with agar medium of $\mathrm{pH} 6.0$ prepared with collection strains were tested for sensitivity to the fluoroquinolones ciprofloxacin and flumequine. The sensitivity of the plate methods to ciprofloxacin was tested by application of $10 \mu \mathrm{L}$ of ciprofloxacin working solution with concentration of $0.3 \mu \mathrm{g} / \mathrm{mL}$, which was used as a positive control in a disc with a diameter of $6 \mathrm{~mm}$. Except for those using $Y$. mollaretii and Y. ruckeri CCM 4620 test strains, all plate methods were sensitive to ciprofloxacin (Table 1). The flumequine sensitivity of the plates with individual testing strains was tested with fortified milk samples with flumequine concentrations $0.5,1,1.5,2,2.5$, and $3 \mathrm{MRL}$ ) by applying a $100 \mu \mathrm{L}$ sample to a $12.7 \mathrm{~mm}$ diameter disc. Of the six strains tested, only the plate method with the $Y$. ruckeri strain CCM 8467 demonstrated sensitivity to flumequine in concentrations of 2, 2.5, and 3 times MRL.

Setting conditions with the $Y$. ruckeri CCM 8467 strain. When setting the method conditions, the Y. ruckeri CCM 8467 strain sensitivity to flumequine was tested in agar with pH 6.0 and 8.0. Fortified milk samples with flumequine concentrations ranging from 1 to 3 times the MRL were investigated in plates with varying test strain cell concentrations. The sensitivity of the plates was also determined by testing $10 \mu \mathrm{L}$ of ciprofloxacin working solution $(c=0.3 \mu \mathrm{g} / \mathrm{mL})$ in a disc with a diameter of $6 \mathrm{~mm}$. Based on the strain growth in agar mediums of different $\mathrm{pH}$ values ( $\mathrm{pH} 6.0$ and 8.0), readability of the inhibition zones, and size of the inhibition zone around the disc with the positive control, the optimal concentration of cells in the agar medium was set at about $9.10^{5}-10^{6} \mathrm{CFU} / \mathrm{mL}$. The mean size of inhibition zone in agar of $\mathrm{pH} 6.0$ for the disc with the positive control (ciprofloxacin) was $6.5 \pm 1.5 \mathrm{~mm}$. The $Y$. ruckeri method in agar of $\mathrm{pH} 8.0$ at flumequine concentration equal to 3 times the MRL did not have sufficient sensitivity, since the size of the inhibition zone was only $0.5 \mathrm{~mm}$. With agar of $\mathrm{pH} 6.0$, the sample was assessed as suspect (inhibition zone size $\geq 2 \mathrm{~mm}$ ) with flumequine concentration of $2 \mathrm{MRL}$. The results shown in Table 2 indicate that the method was more sensitive to flumequine in agar of $\mathrm{pH} 6.0$.

Detection capability. The plates' sensitivity was determined in accordance with the requirements of EC legislation (8) on performance characteristics of screening methods based on detection capability $(C C \beta)$. $C C \beta$ is defined in a clause of the Annex to Commission Decision 2002/657/EC (8). CC $\beta$ is the smallest content of the analyte that may be detected, identified and/or quantified in a sample with an error probability of $\beta$. In the case of analytes with an established MRL, $C C \beta$ is the concentration at which the method is able to detect the permitted limit concentrations with a statistical certainty of $1-\beta$. $C C \beta$ is the concentration at which only $\leq 5 \%$ false compliant results remain. In this case, $C C \beta$ must be less than or equal to the MRL (9). Fortified milk samples with a standard fluoroquinolone concentration were used to determine the detection capability. Each fortified sample with a different basic concentration of the antibiotic $(0.5,1,2,2.5$, and 3 times the MRL) was tested 20 times. The fortified samples with further concentrations of antibiotics for more specific designation of the level of the detection capability were tested 60 times. Testing of the modified method took place in two independent laboratories.

Detection capability determination with the Y. ruckeri CCM 8467 strain to selected fluoroquinolones. Based on the above results, the plate diffusion method using the Y. ruckeri CCM 8467 strain was tested: the concentration of cells in the agar medium was at $9.10^{5}-10^{6}$ $\mathrm{CFU} / \mathrm{mL}$, discs were $12.7 \mathrm{~mm}$ in diameter. Antimicrobial Inhibitor Test Agar had $\mathrm{pH}$ of 6.0, incubation time lasted $18-24 \mathrm{~h}$, and incubation temperature 
Table 1. Sensitivity of the plate methods with Yersinia spp

\begin{tabular}{lllllll}
\hline $\begin{array}{l}\text { Antimicrobial } \\
\text { substance }\end{array}$ & Y. aldovae & Y. bercovieri & Y. mollaretii & Y. rohdei & Y. ruckeri & Y. ruckeri \\
\hline ciprofloxacine & CCM 3754 & CCM 4207 & CCM 4209 & CCM 4075 & CCM 4620 & CCM 8467 \\
\hline
\end{tabular}

Table 2. Setting plate method conditions with Y. ruckeri CCM 8467, agar pH 6.0 and 8.0, testing milk samples with flumequine concentrations of 0.5-3 times the MRL, size of the inhibition zone in mm

\begin{tabular}{llllll}
\hline \multirow{2}{*}{ Agar $\mathrm{pH}$} & \multicolumn{4}{c}{ Flumequine concentration (multiple MRL)/size of the inhibition zone in mm } \\
& 1 MRL & $2.5 \mathrm{MRL}$ & $2.7 \mathrm{MRL}$ & $2.9 \mathrm{MRL}$ & $3 \mathrm{MRL}$ \\
\hline 6.0 & 0 & $3.5 \pm 0.9$ & $3.5 \pm 0.7$ & $4.5 \pm 0.6$ & $4.5 \pm 0.5$ \\
8.0 & 0 & $0.25 \pm 0.2$ & $0.25 \pm 0.2$ & $0.5 \pm 0.3$ & $0.5 \pm 0.3$ \\
\hline
\end{tabular}

Table 3. Detection capability $-C C \beta$ of plate diffusion method with $Y$. ruckeri CCM 8467 for selected fluoroquinolones

\begin{tabular}{llll}
\hline $\begin{array}{l}\text { Antimicrobial } \\
\text { substance }\end{array}$ & $\begin{array}{l}\text { MRL EU } \\
(\mu \mathrm{g} / \mathrm{kg})\end{array}$ & $\begin{array}{l}C C \beta \\
(\mu \mathrm{g} / \mathrm{L})\end{array}$ & $C C \beta / \mathrm{MRL}$ \\
\hline danofloxacin & 30 & 42 & $\mathrm{CC} \beta>\mathrm{MRL}$ \\
marbofloxacin & 75 & 30 & $\mathrm{CC} \beta<\mathrm{MRL}$ \\
flumequine & 50 & 100 & $\mathrm{CC} \beta>\mathrm{MRL}$ \\
enrofloxacin & $100^{* *}$ & 20 & $\mathrm{CC} \beta<\mathrm{MRL}$ \\
ciprofloxacin & 10 & $\mathrm{CC} \beta<\mathrm{MRL}$ \\
\hline
\end{tabular}

**sum of enrofloxacin and ciprofloxacin

was $30^{\circ} \mathrm{C}$. The sensitivity of the method with regard to the determined MRLs for the selected fluoroquinolones is shown in Table 3 . The method is especially sensitive to ciprofloxacin and enrofloxacin. The determined $C C \beta$ for the specified fluoroquinolones was at concentrations of 10 and $20 \mu \mathrm{g} / \mathrm{L}(0.1$ and 0.2 times the MRL). Among other fluoroquinolones, the method is also capable of reliably detecting marbofloxacin. $C C \beta$ is at concentrations lower than the MRL at $30 \mu \mathrm{g} / \mathrm{L}$ ( $0.4 \mathrm{MRL})$. In relation to the MRL, the method is less sensitive to danofloxacin, where the $C C \beta$ value reaches concentrations slightly higher than the stipulated MRL (42 $\mu \mathrm{g} / \mathrm{L}, 1.4 \mathrm{MRL})$. The results in Table 3 also show that $C C \beta$ for flumequine is equal to twice the limit, at $100 \mu \mathrm{g} / \mathrm{L}(2 \mathrm{MRL})$.

\section{Discussion}

Antimicrobial residue examination in animal origin raw materials currently relies on a two-stage testing system based on current EU legislation (8) which defines screening and confirmatory methods. A plate diffusion method is an integral part of the system of antimicrobial residue detection in milk in many countries and it is used mainly as a screening method. The commonly used plate diffusion methods include the Four-Plate Test (FPT) developed in 1980 by Bogaerts and Wolf (5), which is, however, gradually being complemented and replaced by newly developed methods that show higher sensitivity to specific antimicrobial groups. In Europe, the Community Reference Laboratory in Fougères recommends 5-plate diffusion called the STAR method which involves a plate method with E. coli ATCC 11303 showing increased sensitivity to fluoroquinolones for the screening of antimicrobial residues in milk $(14,15)$.

Navrátilová et al. (17) confirmed, in consensus with other published data, that the plate method with
E. coli ATCC 11303 is not sensitive enough to flumequine; its sensitivity to this antimicrobial being insufficient even at concentrations equal to 9 times the MRL. For the other tested fluoroquinolones (danofloxacin, marbofloxacin, ciprofloxacin, and enrofloxacin), the plate method with $E$. coli achieves detection capability $C C \beta$ in concentrations $\leq$ than the MRLs. In the past 20 years, plate methods applying bacteria other than $E$. coli to detect quinolones have been developed, but they have mostly focused on detecting quinolones in other products of animal origin (egg, meat, fish, and organs). The aim of this study was thus the selection of a test strain for a plate diffusion method which would exhibit sensitivity to fluoroquinolones, in particular to flumequine. As reported previously, one of the test strains suitable for quinolone detection is the $Y$. ruckeri strain. The $Y$. ruckeri plate method was developed to detect oxolinic acid in fish muscle (3), later applied to detect other quinolones in eggs and poultry muscle (21), or in meat and organs of slaughter animals (22), but not in milk.

Of the collection strains of Yersinia spp. used to prepare the plate method and tested for sensitivity to ciprofloxacin and flumequine, only one strain, namely Y. ruckeri CCM 8467, demonstrated sensitivity to flumequine. Therefore it was selected as a test strain for the plate diffusion method. The sensitivity of a plate method can be influenced by a variety of factors: composition and $\mathrm{pH}$ of the agar medium, selection of the test strain, test cell concentration in the agar medium, sample application method, sample quantity, $\mathrm{pH}$ of the sample to be examined, incubation period, and temperature. The selected optimum concentration of test strain cells in the agar medium was close to the cell concentration of $10^{6} \mathrm{CFU} / \mathrm{mL}$ which was selected by Pikkemaat et al. (22) in the plate method with Y. ruckeri NCIM 13282 to detect quinolones in muscles and organs. Based on the examination results of the milk 
samples enriched with flumequine, the test agar with $\mathrm{pH}$ 6.0 was selected, which corresponds to the results of a study by Okerman et al. (20). In their view, flumequine detection appears optimal at agar $\mathrm{pH}$ 6.0, not at $\mathrm{pH}$ 8.0.

When examining samples of milk by a plate diffusion method, paper discs of a specific size with standard absorbency are usually used. Alternatively, the application of milk samples may be performed directly into the wells formed in the agar. The method sensitivity was increased by applying a milk sample to a $12.7 \mathrm{~mm}$ disc having the ability to absorb a sample volume of $100 \mu \mathrm{L}$. For example, the STAR method recommends $9 \mathrm{~mm}$ discs that have absorption capacity of $30 \mu \mathrm{L}$ to examine milk. The $Y$. ruckeri strain showed better growth ability at $30^{\circ} \mathrm{C}$, which is consistent with other studies using the $Y$. ruckeri strain as a test strain in a plate diffusion method $(21,22)$.

The method with Y. ruckeri CCM 8467 test strain seems among fluoroquinolones to be very sensitive to ciprofloxacin and enrofloxacin. The sensitivity was comparable with the plate method using E. coli (17), in which the value of $C C \beta$ reached $40 \mu \mathrm{g} / \mathrm{L}$ for enrofloxacin and $20 \mu \mathrm{g} / \mathrm{L}$ for ciprofloxacin, thus lower than 0.5 of MRL (17). The method is also well able to detect marbofloxacin; $C C \beta$ is at concentrations of $30 \mu \mathrm{g} / \mathrm{L}$. In the $E$. coli method, the sensitivity reaches levels slightly lower than the set limit $(70 \mu \mathrm{g} / \mathrm{L} \mathrm{x} 75 \mu \mathrm{g} / \mathrm{kg})$. The results also show that $C C \beta$ for flumequine achieves $100 \mu \mathrm{g} / \mathrm{L}$ (twice the MRL). When compared to the sensitivity of the plate method with $E$. coli which did not show sensitivity even at a concentration of flumequine equal to nine times the MRL, the $Y$. ruckeri method is more sensitive, nevertheless, it is not sensitive enough to meet the requirement for screening methods that should be sensitive to amounts equal to or less than the MRL.

In conclusion, it can be summarised that by determining the detection capability of the plate diffusion method with the Yersinia ruckeri CCM 8467 strain for selected fluoroquinolones, it was found that this method is able to reliably detect the fluoroquinolones ciprofloxacin, enrofloxacin, and marbofloxacin. With regard to the MRL, the method is less sensitive to danofloxacin and flumequine. In the system of antimicrobial substance detection, it is important that the screening method sensitivity to antimicrobial agents is determined at concentrations close to the respective MRL or lower. The plate method with Yersinia ruckeri CCM 8467 provides a sensitivity improvement over the Community Reference Laboratory's recommended method for flumequine screening but does not meet the requirement for screening methods that should be sensitive to amounts equal to or less than the MRL. Further work is necessary to hone the method's sensitivity.

Conflict of Interests Statement: The authors declare that there is no conflict of interests regarding the publication of this article.
Financial Disclosure Statement: The source of funding of this research and article was the research project grant "KUS" QJ1230044 from the NAZV (National Czech Agency of Agricultural Research).

Animal Rights Statement: None required.

\section{References}

1. Aleksic S., Steigerwalt A.G., Bocke-Mühl J., Huntley-Carter G.P., Brenner D.J. Yersinia rohdei sp. nov. isolated from human and dog feces and surface water. Int J Syst Bacteriol 1987, 37, 327-332.

2. Andreu V., Blasco C., Picó Y.: Analytical strategies to determine quinolone residues in food and the environment. Trends Anal Chem 2007, doi: 10.1016/j.trac.200701.010.

3. Barker G.A.: Detection of 4-quinolone residues in rainbow-trout (Oncorhynchus-mykiss Walbaum) muscle using a bioassay. Aquaculture 1994, 127, 83-90, doi: 10.1016/0044-8486(94) 90194-5.

4. Bercovier H., Steigerwalt A.G., Guiyoule A., Huntley-Carter G., Brenner D.J.: Yersinia aldovae (formerly Yersinia enterocoliticalike group X2): a new species of Enterobacteriaceae isolated from aquatic ecosystems. Int J Syst Bacteriol 1984, 34, 166-172, doi: 10.1099/00207713-34-2-166.

5. Bogaerts R., Wolf F.: A standardized method for the detection of residues of anti-bacterial substances in fresh meat - EEC (European Communities). Fleischwirtschaft 1980, 60, 667-675.

6. Botsoglou N.A., Fletouris D.J.: Drug residues and public health. In: Drug residues in foods. Marcel Dekker, New York, 2001, pp. 269-295.

7. Cháfer-Pericás C., Maquieira Á., Puchades R.: Fast screening methods to detect antibiotic residues in food samples. Trac Trend Anal Chem 2010, doi: 10.1016/j.trac.2010.06.004.

8. Commission Decision No 657/2002 of 12 August 2002 implementing Council Directive 96/23/EC concerning the performance of analytical methods and the interpretation of results. OJ 17.8.2002, L 221, 8-36.

9. Community Reference Laboratories Residues: Guidelines for the Validation of Screening Methods for Residues of Veterinary Medicines". 20/1/2010. https://ec.europa.eu/food/sites/food/files/ safety/docs/cs_vet-med-residues_guideline_validation_screening_en.pdf.

10. Commission Regulation (EU) No 37/2010 of 22 December 2009 on pharmacologically active substances and their classification regarding maximum residue limits in foodstuffs of animal origin. OJ 20.1.2010, L 15, 1-72.

11. Ellerbroek L.: The microbiological determination of the quinolone carbonic-acid derivatives enrofloxacin, ciprofloxacin and flumequin. Fleischwirtschaft 1991, 71, 187-189.

12. Ewing W.H., Ross A.J., Brenner D.J., Fanning G.R.: Yersinia ruckeri sp. nov., the Redmouth (RM) Bacterium. Int J Syst Bacteriol 1978, 28, 37-44, doi:10.1099/00207713-28-1-37.

13. Ferrini A.M., Mannoni V., Aureli P.: Combined Plate Microbial Assay (CPMA): a 6-plate-method for simultaneous first and second level screening of antibacterial residues in meat. Food Addit Contam 2006, doi: 10.1080/02652030500307131.

14. Gaudin V., Cadieu N., Sanders P.: STAR Protocol: screening test for antibiotic residues. Version 2, Community Reference Laboratory, Fougères, 2002, pp. 10-11.

15. Gaudin V., Maris P., Fuselier R., Ribouchon J.L., Cadieu N., Rault A.: Validation of a microbiological method: the STAR protocol, a five-plate test, for screening of antibiotic residues in milk. Food Addit Contam A 2004, doi: 10.1080/02652030410001667575.

16. Myllyniemi A.L., Nuotio L., Lindfors E., Rannikko R., Niemi A., Bäckman C.: A microbiological six-plate method for the identification of certain antibiotic groups in incurred kidney and muscle samples. Analyst 2001, doi: 10.1039/B100135N 
17. Navrátilová P., Vyhnálková J., Vorlová L., Jeřábková J.: A plate diffusion method for detecting fluoroquinolone residues in raw cow's milk. Czech J Food Sci 2014, 32, 260-264.

18. Nouws J.F.M., Van Egmond H., Smulders I., Loeffen G., Schouten J., Stegeman H.: A microbiological assay system for assessment of raw milk exceeding EU maximum residue levels. Int Dairy J 1999, doi: 10.1016/S0958-6946(99)00026-6.

19. Okerman L., Croubels S., De Baere S., Van Hoof J., De Backer P., De Brabander H.: Inhibition tests for detection and presumptive identification of tetracyclines, beta-lactam antibiotic and quinolones in poultry meat. Food Addit Contam 2001, doi: $10.1080 / 02652030120410$.

20. Okerman L., Noppe H., Cornet V., De Zutter L.: Microbiological detection of 10 quinolone antibiotic residues and its application to artificially contaminated poultry samples. Food Addit Contam 2007, 24, 252-257, doi: 10.1080/02652030600988020.
21. Pikkemaat M.G., Mulder P.P.J., Elferink J.W.A., De Cocq A., Nielen M.W.F., Van Egmond H.J.: Improved microbial screening assay for the detection of quinolone residues in eggs and poultry muscle. Food Addit Contam A 2007, doi: 10.1080/02652030701295275.

22. Pikkemaat M.G., Van Dijk O.S., Schouten J., Rapallini M., Van Egmond H.J.: A new microbial screening method for the detection of antimicrobial residues in slaughter animals: the Nouws antibiotic test (NAT-screening). Food Control 2008, doi: 10.1016/j.foodcont.2007.08.002.

23. Pikkemaat M.G.: Microbial screening methods for detection of antibiotic residues in slaughter animals. Anal Bioanal Chem 2009, 395, 893-905, doi: 10.1007/s00216-009-2841-6.

24. Wauters G., Janssens M., Steigerwalt A.G., Brenner D.J.: Yersinia mollaretii sp. nov. and Yersinia bercovieri sp. nov., formerly called Yersinia enterocolitica biogroups 3A and 3B. Int J Syst Bacteriol 1988, 38, 424-429, doi: 10.1099/00207713-38-4-424. 